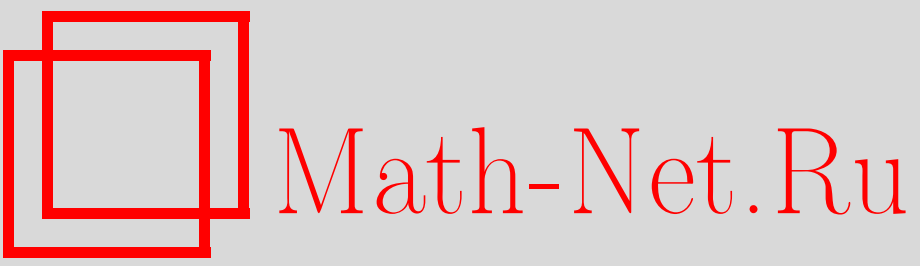

Б. М. Зупник, Связи для суперпотенциалов в гармонических калибровочных теориях с восемью суперзарядами, ТМФ, 1999, том 120, номер 2, 324-331

DOI: https://doi.org/10.4213/tmf779

Использование Общероссийского математического портала Math-Net.Ru подразумевает, что вы прочитали и согласны с пользовательским соглашением

http://www.mathnet.ru/rus/agreement

Параметры загрузки:

IP: 54.174 .149 .18

26 апреля 2023 г., 14:00:41 


\section{СВЯЗИ ДЛЯ СУПЕРПОТЕНЦИАЛОВ В ГАРМОНИЧЕСКИХ КАЛИБРОВОЧНЫХ ТЕОРИЯХ С ВОСЕМЬЮ СУПЕРЗАРЯДАМИ}

Рассматриваются $D$-мерные суперсимметричные калибровочные теории с восемью суперзарядами $(D \leqslant 6, \mathcal{N}=8)$ в рамках гармонических суперпространств. Эффективное абелево низкоэнергетическое действие для $D=5$ содержит свободный член и член Черна-Саймонса. Эффективные $\mathcal{N}=8$ суперполевые действия для $D \leqslant 4$ могут быть выражены через суперпотенциалы, удовлетворяющие суперполевым связям и $(6-D)$-мерным уравнениям Лапласа. Обсуждается роль альтернативных гармонических структур.

\section{1. ВВЕДЕНИЕ}

Концепция гармонического суперпространства (HSS) была впервые введена для внемассового описания материальных, калибровочных и гравитационных суперполевых теорий с $D=4, N_{4}=2$ суперсимметрией $[1,2]$. HSS-подход использовался также для последовательного описания гипермультиплетов и векторного мультиплета с $D=6$, $N_{6}=1$ суперсимметрией [3]. Для классификации этих моделей в различных размерностях вместо числа спинорных представлений для суперзарядов $N_{D}$ удобно использовать полное число суперзарядов $\mathcal{N}=8$. Универсальность гармонических суперпространств связана с построением $\mathcal{N}=8$ моделей для размерностей $D<6$ посредством размерной редукции. HSS-действия для гипермультиплетов $q^{+}, \omega$ и препотенциала Янга-Миллса $V^{++}$описываются универсальными формулами во всех размерностях $D \leqslant 6$. Тем не менее $\mathcal{N}=8$ суперсимметрии имеют некоторые специфические свойства в каждой размерности, связанные с различиями в структуре групп Лоренца $L_{D}$, групп максимальных автоморфизмов $R_{D}$ и множеств центральных зарядов $Z_{D}$. В частности, альтернативные HSS-структуры были найдены для случаев $D=2$ [4] и $D=3$ [5]. Мы будем изучать HSS-конструкции низкоэнергетических эффективных действий для абелевых калибровочных супермультиплетов в размерностях $D=1,2,3,5$ с помощью аналитического препотенциала $V^{++}$и $(6-D)$-компонентной напряженности $W\left(V^{++}\right)$, удовлетворяющей суперполевым связям. Аналогичные проблемы обсуждались ранее в рам-

*Объединенный институт ядерных исследований, Лаборатория теоретической физики им. Н. Н. Боголюбова, Дубна, Московская обл., Россия. E-mail: zupnik@thsun1.jinr.dubna.su 
ках компонентного полевого формализма или формализма с $\mathcal{N}=4, D=1,2,3$ суперполями (см. [6-9]).

Основным результатом этой работы является построение в полном $\mathcal{N}=8$ суперпространстве для размерностей $D=1,2,3,5$ кулоновских эффективных действий, которые содержат произведение гармонических связностей и суперпотенциалов $f_{D}(W)$, удовлетворяющих $(6-D)$-мерным уравнениям Лапласа.

Взаимодействия суперполей с восемью суперзарядами имеют универсальные и специфические свойства в различных размерностях, однако решение большинства геометрических и динамических проблем упрощается в HSS-форомализме. (6 - D)-мерные уравнения Лапласа для низкоэнергетических суперпотенциалов вытекают из калибровочной инвариантности в HSS. Теоремы об отсутствии перенормировки связаны с поиском $R_{D}$-инвариантных решений этих уравнений. Анализ уравнений для суперпотенциалов связан с существованием альтернативных HSS-структур, используюших различные типы гармоник в $D \leqslant 3, \mathcal{N}=8$ теориях, которые адекватно отражают свойства преобразований дуальности в этих моделях.

\section{2. ЭФФЕКТИВНЫЕ ДЕЙСТВИЯ В ПОЛНЫХ И АНАЛИТИЧЕСКИХ СУПЕРПРОСТРАНСТВАХ}

2.1. Рассмотрим кратко гармоническое суперпространство с $D=5, \mathcal{N}=8$ суперсимметрией. Общее 5 -мерное $(5 D)$ суперпространство имеет координаты $z=\left(x^{\mathbf{m}}, \theta_{i}^{\alpha}\right)$, где $\mathbf{m}$ и $\alpha-5$-векторные и 4-спинорные индексы группы Лоренца $L_{5}=S O(4,1)$, соответственно, а $i-2$-спинорньй индекс группы автоморфизмов $R_{5}=S U(2)$. Антисимметричные $5 D \Gamma$-матрицы $\left(\Gamma_{\mathbf{m}}\right)_{\alpha \gamma}$ и инвариантная симплектическая матрица $\Omega_{\alpha \rho}$ могут быть выражены через матрицы Вейля и $\varepsilon$-символы в $S L(2, C)$.

Удобно использовать биспинорные представления $5 D$ координат и частных производHыx

$$
x^{\alpha \rho}=\frac{1}{2}\left(\Gamma_{\mathbf{m}}\right)^{\alpha \rho} x^{\mathbf{m}}, \quad \partial_{\alpha \rho}=\frac{1}{2}\left(\Gamma^{\mathbf{m}}\right)_{\alpha \rho} \partial_{\mathbf{m}}
$$

Основные соотношения между спинорными производными $D_{\alpha}^{k}$ в обшем $D=5, \mathcal{N}=8$ суперпространстве имеют следуюший вид:

$$
\left\{D_{\alpha}^{k}, D_{\gamma}^{l}\right\}=i \varepsilon^{k l}\left(\partial_{\alpha \gamma}+\frac{1}{2} \Omega_{\alpha \gamma} Z\right),
$$

где $Z$ - вещественньй центральньй заряд.

$R_{5}$-инвариантные проекции спинорных производных $D_{\alpha}^{ \pm}=u_{i}^{ \pm} D_{\alpha}^{i}$ и координат гармонического суперпространства $\zeta=\left(x_{A}^{\mathbf{m}}, \theta^{\alpha+}, \theta^{\alpha-}\right)$ могут быть определены с помошью $S U(2) / U(1)$-гармоник $u_{i}^{ \pm}$по аналогии с тем, как это сделано в работе [1] $(i-2$-спинорный индекс $S U(2)$ и $q= \pm 1-U(1)$-заряд). Аналитический абелев препотенциал $V^{++}(\zeta, u)$ описывает $5 D$ векторный супермультиплет, который включает вешественное скалярное поле $\phi$, максвелловское поле $A_{\mathbf{m}}$, изодублет 4 -спиноров $\lambda_{i}^{\alpha}$ и вспомогательный изотриплет $X^{i k}$. Вешественное скалярное суперполе этой теории выражается 
через гармоническую связность с $U(1)$-зарядом -2 :

$$
W=-\frac{i}{2} D^{+\alpha} D_{\alpha}^{+} \int d u_{1} \frac{V^{++}\left(x, u_{1}\right)}{\left(u^{+} u_{1}^{+}\right)^{2}}=-2 i D^{(+2)} V^{--},
$$

где $\left(u^{+} u_{1}^{+}\right)^{-2}$ - гармоническая обобшенная функция [2]. Эти суперполя удовлетворяют следуюшим связям:

$$
\begin{gathered}
D^{++} V^{--}=D^{--} V^{++}, \quad D^{ \pm \pm} W=0, \\
D_{\alpha \rho}^{(+2)} W=0, \quad D_{\alpha \rho}^{(+2)}=D_{\alpha}^{+} D_{\rho}^{+}-\frac{1}{4} \Omega_{\alpha \rho} D^{+\sigma} D_{\sigma}^{+} .
\end{gathered}
$$

Нетрудно построить наиболее общее низкоэнергетическое $U(1)$-калибровочное действие в полном $D=5, \mathcal{N}=8$ гармоническом суперпространстве

$$
S_{5}=\int d^{5} x d^{8} \theta d u V^{++} V^{--}\left[g_{5}^{-2}+k_{5} W\right]
$$

где $g_{5}$ - константа связи размерности $1 / 2$, а $k_{5}$ - безразмерная константа $5 D$ взаимодействия Черна-Саймонса.

Низкоэнергетическое $D=4, \mathcal{N}=8$ эффективное действие сохраняет группу автоморфизмов $S U(2)$ и нарушает $U_{R}(1)$-симметрию. Соответствующий $D=4$ суперпотенциал $f(W, \bar{W})=[F(W)+\bar{F}(\bar{W})]$ удовлетворяет $2 D$ уравнению Лапласа, которое имеет только голоморфные и антиголоморфные решения.

2.2. Аналогичная $D=3, \mathcal{N}=8$ калибровочная теория может быть построена в суперпространстве с группой автоморфизмов $R_{3}=S U_{l}(2) \times S U_{r}(2)$. Рассмотрим координаты обшего суперпространства $z=\left(x^{\alpha \beta}, \theta_{i a}^{\alpha}\right)$, где используются двухкомпонентные индексы: $(\alpha, \beta, \ldots)$ для пространственно-временной групшы $S L(2, R),(i, k, \ldots)$ для группы $S U_{l}(2),(a, b, \ldots)$ для группы $S U_{r}(2)$. Соотношения между спинорными производными имеют вид

$$
\left\{D_{\alpha}^{k a}, D_{\beta}^{l b}\right\}=i \varepsilon^{k l} \varepsilon^{a b} \partial_{\alpha \beta}+i \varepsilon^{k l} \varepsilon_{\alpha \beta} Z^{a b},
$$

где $\partial_{\alpha \beta}=\partial / \partial x^{\alpha \beta}$, а $Z^{a b}$ - центральные заряды, которые коммутируют со всеми генераторами, за исключением генераторов $S U_{r}(2)$.

Мы будем использовать обозначения $u_{i}^{ \pm} \equiv u_{i}^{( \pm 1,0)}$ для гармоник группы $S U_{l}(2)$ и $v_{a}^{(0, \pm 1)}$ для $S U_{r}(2)$-гармоник, а также обозначения $D_{l}^{ \pm \pm}$для $l$-гармонических производных и $V_{l}^{ \pm \pm}$для $l$-формы $3 D$ гармонических калибровочных суперполей [5]. Обозначения с двумя $U(1)$-зарядами будут применяться для бигармонических суперполей. Калибровочно-ковариантное $S U_{r}(2)$-биспинорное суперполе $D=3, \mathcal{N}=8$ калибровочной теории содержит соответствующую гармоническую связность [5]

$$
W^{a b}=-i D^{+\alpha a} D_{\alpha}^{+b} V_{l}^{--},
$$

где $D_{\alpha}^{+b}=u_{i}^{+} D_{\alpha}^{i b}$. Это суперполе не зависит от гармоник в абелевом случае. 
$S L(2, R) \times S U_{l}(2)$-инвариантное кулоновское эффективное действие может быть выражено через суперпотенциал $f_{3}\left(W^{a b}\right)$ :

$$
S_{3}=\int d^{3} x d^{8} \theta d u V_{l}^{++} V_{l}^{--} f_{3}\left(W^{a b}\right) .
$$

Калибровочная инвариантность накладывает следуюшее условие связи:

$$
D_{\alpha}^{+c} D_{c \beta}^{+} f_{3}\left(W^{a b}\right)=0 .
$$

Эта связь эквивалентна трехмерному уравнению Лапласа

$$
\Delta_{3}^{w} f_{3}\left(W^{a b}\right)=0, \quad \Delta_{3}^{w}=\frac{\partial}{\partial W^{a b}} \frac{\partial}{\partial W_{a b}} .
$$

$R_{3}$-инвариантное решение уравнения (11) имеет вид

$$
f_{3}^{R}\left(w_{3}\right)=g_{3}^{-2}+k_{3} w_{3}^{-1}, \quad w_{3}=\sqrt{W^{a b} W_{a b}},
$$

где $g_{3}$ - константа связи размерности $d=-1 / 2$, а $k_{3}$ - безразмерная константа $\mathcal{N}=8$ взаимодействия типа Весса-Зумино-Новикова-Виттена для векторного мультиплета. Это взаимодействие хорошо определено для ненулевых значений модуля центральных зарядов $|Z|_{3}=\sqrt{Z^{a b} Z_{a b}}$, когда его разложение по $\widehat{W}_{a b}=W_{a b}-Z_{a b}$ несингулярно. Следует отметить, что суперполевые взаимодействия $3 D$ векторных мультиплетов с безразмерными константами (члены Черна-Саймонса) были построены ранее для случаев $\mathcal{N}=4[10]$ и $\mathcal{N}=6[11,5]$.

Связи для суперполя $W^{a b}$ можно интерпретировать как альтернативную $r$-аналитичность следующей проекции этого суперполя в бигармоническом суперпространстве:

$$
\begin{aligned}
& W^{(0,2)}=v_{a}^{(0,1)} v_{b}^{(0,1)} W^{a b}=-i D^{(2,2)} V_{l}^{(-2,0)}(x, u)=-i \int d u D^{(-2,2)} V_{l}^{(2,0)}(x, u), \\
& \text { где } V_{l}^{( \pm 2,0)} \equiv V_{l}^{ \pm \pm} \text {и } D^{( \pm 2,2)}=u_{i}^{( \pm 1,0)} u_{k}^{( \pm 1,0)} v_{a}^{(0,1)} v_{b}^{(0,1)} D^{i a \alpha} D_{\alpha}^{k b} .
\end{aligned}
$$

$3 D$ суперпотенциал (11) можно представить в виде интеграла по гармоникам $v_{a}^{(0, \pm 1)}$ :

$$
f_{3}\left(W^{a b}\right)=\int d v F_{3}\left[W^{(0,2)}, v^{(0, \pm 1)}\right]
$$

где $F_{3}$ - произвольная функция с $q=(0,0)$. Эффективное действие в полном суперпространстве (9) можно преобразовать в эквивалентное представление в $r$-аналитическом суперпространстве:

$$
\begin{gathered}
S_{3}=\int d^{3} x D^{(0,-4)} d v\left[W^{(0,2)}\right]^{2} F_{3}\left[W^{(0,2)}, v^{(0, \pm 1)}\right], \\
D^{(0,-4)}=D^{(2,-2)} D^{(-2,-2)} .
\end{gathered}
$$

Зеркальная симметрия связывает $l$-векторный мультиплет $W^{(0,2)}\left(V^{(2,0)}\right)$ с $r$-аналитическим гипермультиплетом $\omega_{r}$. 
2.3. Двумерные $(4,4)$ суперполя и соответствующие $\sigma$-модели обсуждались для обычного суперпространства в $[12,13]$ и в рамках альтернативных гармонических суперпространств в [4]. Калибровочная теория $(4,4)$ рассматривалась в формализме $(2,2)$ суперпространства [7]. Мы будем изучать геометрию этой теории в рамках явно ковариантного гармонического формализма. В $(4,4)$ суперпространстве с координатами $\left(y, \bar{y}, \theta^{i \alpha}, \bar{\theta}^{i a}\right)$ будет использоваться группа автоморфизмов $R_{2}=S U_{c}(2) \times S U_{l}(2) \times$ $S U_{r}(2)$ (соответствуюшие обозначения спинорных индексов для этих групп: $c-i, k, \ldots$; $l-\alpha, \beta, \ldots ; r-a, b, \ldots)$. Алгебра спинорных производных этого суперпространства

$$
\begin{aligned}
& \left\{D_{k \alpha}, D_{l \beta}\right\}=\varepsilon_{k l} \varepsilon_{\alpha \beta} \partial_{y}, \\
& \left\{\bar{D}_{k a}, \bar{D}_{l b}\right\}=\varepsilon_{k l} \varepsilon_{a b} \bar{\partial}_{y}, \\
& \left\{D_{k \alpha}, \bar{D}_{l b}\right\}=i \varepsilon_{k l} Z_{\alpha b}
\end{aligned}
$$

включает центральные заряды $Z_{\alpha b}$.

Суперполевые связи неабелевой $(4,4)$ калибровочной теории имеют вид

$$
\begin{aligned}
& \left\{\nabla_{k \alpha}, \nabla_{l \beta}\right\}=\varepsilon_{k l} \varepsilon_{\alpha \beta} \nabla_{y}, \\
& \left\{\bar{\nabla}_{k a}, \bar{\nabla}_{l b}\right\}=\varepsilon_{k l} \varepsilon_{a b} \bar{\nabla}_{y}, \\
& \left\{\nabla_{k \alpha}, \bar{\nabla}_{l b}\right\}=i \varepsilon_{k l} W_{\alpha b} .
\end{aligned}
$$

В работе [4] обсуждаются три типа гармоник: $u_{i}^{ \pm}=u^{( \pm 1,0,0)}$ для $S U_{c}(2) / U_{c}(1)$, $l_{\alpha}^{(0, \pm 1,0)}$ для $S U_{l}(2) / U_{l}(1)$ и $r_{a}^{(0,0, \pm 1)}$ для $S U_{r}(2) / U_{r}(1)$ (в наших обозначениях). Основные геометрические структуры калибровочной теории связаны главным образом с гармониками $u_{i}^{ \pm}$и соответствующими аналитическими координатами $\zeta_{c}=\left(y_{c}, \theta^{+\alpha}\right)$ и $\bar{\zeta}_{c}=\left(\bar{y}_{c}, \bar{\theta}^{+a}\right) . S O(4)$-векторная суперполевая напряженность $w_{m}$ для $2 D$ аналитического калибровочного препотенциала $V_{c}^{++}\left(\zeta_{c}, \bar{\zeta}_{c}, u\right)$ (c-векторный мультиплет) может быть построена по аналогии со случаем $D=3$ :

$$
W_{\alpha b} \equiv\left(\sigma^{m}\right)_{\alpha b} W_{m}=-i D_{\alpha}^{+} \bar{D}_{b}^{+} V_{c}^{--},
$$

где $\left(\sigma^{m}\right)_{\alpha b}-S O(4)$-матрицы Вейля. $W_{\alpha b}$ удовлетворяет суперполевым связям, аналогичным связям так называемого твистованного мультиплета [12].

В полном $(4,4)$ суперпространстве можно построить эффективное действие $U(1)$-калибровочной теории:

$$
S_{2}=\int d^{2} x d^{8} \theta d u V_{c}^{++} V_{c}^{--} f_{2}\left(W_{m}\right), \quad\left(D^{+}\right)^{2} f_{2}\left(W_{m}\right)=\left(\bar{D}^{+}\right)^{2} f_{2}\left(W_{m}\right)=0 .
$$

Общий $(4,4)$ суперпотенциал удовлетворяет $4 D$ уравнению Лапласа

$$
\Delta_{4}^{w} f_{2}\left(W_{m}\right)=0, \quad \Delta_{4}^{w}=\frac{\partial}{\partial W_{m}} \frac{\partial}{\partial W_{m}} .
$$

$R_{2}$-инвариантное решение для $(4,4)$ суперпотенциала определяется однозначно:

$$
f_{2}^{R}\left(w_{2}\right)=g_{2}^{-2}+k_{2} w_{2}^{-2}, \quad w_{2}=\sqrt{W_{m} W_{m}} .
$$


Аналогичная функция рассматривалась при изучении $R_{2}$-инвариантного $(2,2)$ кэлерова потенциала $D=2$ калибровочной теории $(4,4)[7]$. Явно $(4,4)$ ковариантный формализм гармонической калибровочной теории упрощает доказательство теоремы об отсутствии перенормировки.

Бигармоническое представление $(4,4)$ суперпотенциала естественно для решений уравнения (24)

$$
f_{2}\left(W^{\alpha a}\right)=\int d l d r F_{2}\left[W^{(0,1,1)}, l, r\right], \quad W^{(0,1,1)}=l_{\alpha}^{(0,1,0)} r_{a}^{(0,0,1)} W^{\alpha a}
$$

где $F_{2}$ - вешественная $r l$-аналитическая функция с нулевыми $U(1)$-зарядами.

Эта проекция векторного мультиплета (22) удовлетворяет условиям $r l$-аналитичности в тригармоническом суперпространстве

$$
\begin{aligned}
& u_{i}^{( \pm 1,0,0)} l_{\alpha}^{(0,1,0)} D^{i \alpha} W^{(0,1,1)} \equiv D^{( \pm 1,1,0)} W^{(0,1,1)}=0 \\
& u_{i}^{( \pm 1,0,0)} r_{a}^{(0,0,1)} \bar{D}^{i a} W^{(0,1,1)} \equiv \bar{D}^{( \pm 1,0,1)} W^{(0,1,1)}=0
\end{aligned}
$$

и гармоническим условиям

$$
D_{c}^{( \pm 2,0,0)} W^{(0,1,1)}=D_{l}^{(0,2,0)} W^{(0,1,1)}=D_{r}^{(0,0,2)} W^{(0,1,1)}=0,
$$

которые аналогичны связям для суперполя $q^{(1,1)}$, полученным в работе [4] (в этом обозначении не указан $U_{c}(1)$-заряд). Отметим, что векторный мультиплет $W^{(0,1,1)}$ coдержит $2 D$ векторное поле вместо вспомогательной скалярной компоненты суперполя $q^{(1,1)}$.

Используя уравнения (26) и (23), можно получить следующее эквивалентное представление эффективного $(4,4)$ действия в $r l$-аналитическом суперпространстве:

$$
\begin{aligned}
S_{2}= & \int d l d r d^{2} x D^{(1,-1,0)} D^{(-1,-1,0)} \bar{D}^{(1,0,-1)} \times \\
& \times \bar{D}^{(-1,0,-1)}\left[W^{(0,1,1)}\right]^{2} F_{2}\left[W^{(0,1,1)}, l, r\right],
\end{aligned}
$$

где

$$
W^{(0,1,1)}=-i D^{(1,1,0)} \bar{D}^{(1,0,1)} V^{(-2,0,0)}=-i \int d u D^{(-1,1,0)} \bar{D}^{(-1,0,1)} V^{(2,0,0)} .
$$

Действие $q^{(1,1)}$ мультиплета с аналогичной структурой $(4,4) \sigma$-модели построено в [4]. Этот мультиплет дуален $r l$-аналитическому мультиплету $\omega^{( \pm 1, \mp 1)}$. 
2.4. Одномерные $\sigma$-модели рассматривались в $\mathcal{N}=4$ суперпространстве $[14,15]$. Это суперпространство использовалось также для доказательства теоремы об отсутствии перенормировки в $\mathcal{N}=8$ калибровочной теории [6].

Мы будем рассматривать $D=1, \mathcal{N}=8$ суперпространство, которое основано на группе автоморфизмов $R_{1}=S U_{c}(2) \times \operatorname{Spin}(5)$ и имеет координаты $\left(t, \theta_{i}^{\alpha}\right)$, где $i-2$-спинорный индекс, а $\alpha-4$-спинорный индекс группы $\operatorname{Spin}(5)=U \mathrm{Sp}(4)$. Алгебра спинорных производных

$$
\left\{D_{\alpha}^{k}, D_{\rho}^{l}\right\}=i \varepsilon^{k l} \Omega_{\alpha \rho} \partial_{t}+i \varepsilon^{k l} Z_{\alpha \rho}
$$

включает центральные заряды $Z_{\alpha \rho}$.

Связи для $1 D$ векторного мультиплета соответствуют условиям интегрируемости для $c$-аналитичности. Аналитические $1 D$ координаты $\zeta_{c}=\left(t_{c}, \theta^{+\alpha}\right)$ можно определить через стандартные гармоники $u_{i}^{ \pm} \equiv u_{i}^{( \pm 1,0,0)}$. Обозначения и алгебра гармонизованных спинорных производных $D_{\alpha}^{ \pm}$подобны для случаев $D=1$ и $D=5$. Суперполевая напряженность для соответствуюших гармонических калибровочных связностей $V_{c}^{ \pm \pm}$представляет собой 5 -вектор $W_{\mathbf{m}}$ (или бесследовый биспинор $\left.W_{\alpha \rho}\right)$ по отношению к $\operatorname{Spin}(5)$ :

$$
W_{\alpha \rho} \equiv \frac{1}{2}\left(\Gamma^{\mathbf{m}}\right)_{\alpha \rho} W_{\mathbf{m}}=-i D_{\alpha \rho}^{(+2)} V_{c}^{--}, \quad D^{(+2)} W_{\alpha \rho}=0
$$

где использованы $\operatorname{Spin}(5)$ Г-матрицы и обозначения (5).

В абелевой калибровочной группе четыре компоненты этого биспинора являются твистованными суперполями, например $D_{1}^{ \pm} W_{13}=D_{3}^{ \pm} W_{13}=0$.

Эффективное действие в полном суперпространстве $D=1$ имеет следующий вид:

$$
S_{1}=\int d t d^{8} \theta d u V_{c}^{++} V_{c}^{--} f_{1}\left(W_{\mathbf{m}}\right) .
$$

Калибровочная инвариантность $S_{1}$ эквивалентна 5-мерному уравнению Лапласа для суперпотенциала

$$
D^{(+2)} f_{1}\left(W_{\mathbf{m}}\right)=0 \longrightarrow \Delta_{5}^{w} f_{1}\left(W_{\mathbf{m}}\right)=0 .
$$

$R_{1}$-инвариантный 1-мерный суперпотенциал

$$
f_{1}^{R}\left(w_{1}\right)=g_{1}^{-2}+k_{1} w_{1}^{-3}
$$

выражается через длину 5-вектора:

$$
w_{1}=\left(W^{\rho \sigma} W_{\rho \sigma}\right)^{1 / 2} .
$$

Отметим, что подобная функция определяет кэлеров потенциал $D=1$ калибровочной теории в $\mathcal{N}=4$ суперполевом формализме [6].

Бигармоническая конструкция обшего $5 D$ суперпотенциала (35) требует использования гармоник $v_{\alpha}^{(0, \pm 1,0)}, v_{\alpha}^{(0,0, \pm 1)}$ группы $U \mathrm{Sp}(4)[16]$ и соответствующей гармонической проекции биспинорного суперполя

$$
\begin{aligned}
f_{1}\left(W^{\alpha \rho}\right) & =\int d v F_{1}\left[W^{(0,1,1)}, v_{\alpha}\right] \\
W^{(0,1,1)} & =v_{\alpha}^{(0,1,0)} v_{\rho}^{(0,0,1)} W^{\alpha \rho}
\end{aligned}
$$


где рассматривается вещественная функция $F_{1}$ суперполя $W^{(0,1,1)}$ и $v$-гармоник. Связи для суперполя $W^{\alpha \rho}$ эквивалентны условиям $v$-аналитичности

$$
u_{i}^{( \pm 1,0,0)} v_{\alpha}^{(0,1,0)} D^{i \alpha} W^{(0,1,1)}=u_{i}^{( \pm 1,0,0)} v_{\alpha}^{(0,0,1)} D^{i \alpha} W^{(0,1,1)}=0
$$

совместно с гармоническими условиями по $u$ - и $v$-переменным

$$
\begin{aligned}
D_{c}^{( \pm 2,0,0)} W^{(0,1,1)} & =D_{v}^{(0,2,0)} W^{(0,1,1)}=0, \\
D_{v}^{(0,0,2)} W^{(0,1,1)} & =D_{v}^{(0,1,1)} W^{(0,1,1)}=0 .
\end{aligned}
$$

По аналогии со случаями $D=2,3$ мы можем анализировать эквивалентную форму $S_{1}$ в $v$-аналитическом суперпространстве и соответствуюшие преобразования дуальности.

Благодарности. Автор признателен Е. А. Иванову за стимулирующие обсуждения.

Работа частично поддержана грантами РФФИ № 96-02-17634, РФФИ-ННИО № 96-02-00180, INTAS № 93-127-ext и INTAS № 96-0308, а также грантом Узбекского фонда фундаментальных исследований № 11/97.

\section{Список литературы}

[1] A. Galperin, E. Ivanov, S. Kalitzin, V. Ogievetsky, E. Sokatchev. Class. Quantum Gravit. 1984. V. 1. P. 469.

[2] A. Galperin, E. Ivanov, V. Ogievetsky, E. Sokatchev. Class. Quantum Gravit. 1985. V. 2. P. 601.

[3] Б. М. Зупник. ЯФ. 1986. Т. 44. C. 793; B. M. Zupnik. Phys. Lett. В. 1987. V. 183. Р. 175.

[4] E. Ivanov, A. Sutulin. Nucl. Phys. B. 1994. V. 432. P. 246; Class. Quantum Gravit. 1997. V. 14. P. 843.

[5] B. M. Zupnik. Harmonic superspaces for three-dimensional theories, hep-th/9804167.

[6] D.-E. Diaconescu, $R$. Entin. A non-renormalization theorem for the $d=1, N=8$ vector multiplet, hep-th/9706059.

[7] D.-E. Diaconescu, N. Seiberg. J. High Ener. Phys. 1997. V. 7. P. 1.

[8] O. Aharony, A. Hanany, K. Intriligator, N. Seiberg, M. J. Strassler. Nucl. Phys. B. 1997. V. 499. P. 67.

[9] N. Seiberg. Phys. Lett. B. 1997. V. 388. P. 753.

[10] Б. М. Зупник, Д. Г. Пак. ТМФ. 1988. Т. 77. С. 97.

[11] Б. М. Зупник, Д. В. Хецелиус. ЯФ. 1988. Т. 47. С. 1147.

[12] S. J. Gates, C. M. Hull, M. Roček. Nucl. Phys. B. 1984. V. 248. P. 157.

[13] O. Gorovoy, E. Ivanov. Nucl. Phys. B. 1992. V. 381. P. 394.

[14] V. P. Berezovoj, A. I. Pashnev. Class. Quantum Gravit. 1991. V. 8. P. 2141.

[15] E. A. Ivanov, A. V. Smilga. Phys. Lett. B. 1991. V. 257. P. 79.

[16] E. Ivanov, S. Kalitzin, Nguen Ai Viet, V. Ogievetsky. J. Phys. A. 1985. V. 18. P. 3433. 